\title{
Expectations from the assumption of hadron-hadron collisions for high energy neutrinos
}

\author{
Carlo Mascaretti* \\ Gran Sasso Science Institute \\ E-mail: carlo.mascaretti@gssi.it \\ Francesco Vissani \\ Gran Sasso Science Institute, INFN, Laboratori Nazionali del Gran Sasso \\ E-mail: francesco.vissani@lngs.infn.it
}

The IceCube collaboration has discovered a new, cosmic component of high-energy neutrinos. Although neutrino oscillations suggest that the cosmic neutrino spectrum is almost the same for every neutrino flavor, the attempts to reconstruct it, based on different analyses, lead to different energy spectra below $100 \mathrm{TeV}$. In this work, we propose a phenomenological model that, assuming collisions between cosmic rays and hadrons as the production mechanism of high-energy neutrinos, yields quantitative expectations for each neutrino flavor. We discuss the detectability of the prompt component of the atmospheric neutrino spectrum, pointing out the most relevant dataset, which has to be muon-neutrino depleted and to cover the energy region $10-100 \mathrm{TeV}$. We argue that the prompt component can cause the spectral difference between the High-EnergyStarting-Event (HESE) and the through-going muon datasets. Finally, we point out the need for adopting a consistent model for the interpretation of the data, stressing that a separate treatment of the different datasets is, by converse, a suboptimal procedure.

36th International Cosmic Ray Conference -ICRC2019-

July 24th - August 1st, 2019

Madison, WI, U.S.A.

${ }^{*}$ Speaker. 


\section{Introduction}

The IceCube detector has provided unique and most important observations of neutrino events with energies ranging between $100 \mathrm{GeV}$ and $10 \mathrm{PeV}$, resulting in the detection of an astrophysical component of neutrinos [1,2] and in the measurement of the atmospheric electron and muon neutrino spectra [3, 4]. Prompt neutrinos, expected from the decay of charmed mesons in the atmosphere, have not been measured yet.

The accepted set of assumptions on the astrophysical component, that are adopted for the interpretation of these findings, includes: 1) isotropy, 2) standard three-flavor oscillations, and 3) an unbroken power law for the energy spectrum of the new component. However, the cosmic neutrino spectrum resulting from the HESE analysis [5] is different from that obtained in the through-going muons analysis [2]. Moreover, if the spectrum from HESE were extrapolated down to energies lower than $100 \mathrm{TeV}$, it would overshoot the gamma-ray diffuse measurements [6].

In this situation, we decided to rely on theoretical guidance. In this work a primary cosmic-ray flux is defined to numerically compute the atmospheric neutrino spectrum. The cosmic neutrino flux is modeled as a single-population power law, assuming $p p$ collisions in dense sources as the production mechanism. The ensuing expectations from theory are combined to the throughgoing muon analysis to make the astrophysical muon neutrino flux phenomenologically precise. The same muon neutrino flux is also used to predict the electron and tau cosmic neutrino fluxes. Credible regions are computed for all neutrino fluxes, which are in turn compared to the available measurements.

Finally, we comment on the results and address 1) the compelling issue of the low-energy softness of the HESE spectrum, 2) the lack of detection of prompt neutrinos in the currently examined datasets, and 3) the methodological consistency of independent analyses of the various datasets.

\section{The expected neutrino fluxes}

\subsection{Atmospheric neutrinos}

Atmospheric neutrinos can be predicted from the observed flux of cosmic rays and the theory of strong interactions. Many (semi-)analytical computations [7, 8, 9] have been carried out, adopting different primary cosmic-ray and hadronic interaction models, in order to predict the conventional component, which results from the decay of pions, kaons, and unflavored mesons, and the prompt component, which comes from charmed meson decays - even though the latter contribution is considerably more uncertain and, moreover, undetected at present.

Following [10], we compute the atmospheric neutrino flux with MCEq [11], adopting the most recent 2.3c version of SYBILL [12], and a primary CR flux defined as follows:

- only protons and helium nuclei are considered, as they are the most abundant elemental species as well as the most relevant, as nuclei of mass number $A$ and energy $E$ produce neutrinos with average energy $E / 20 A$ when colliding with other nuclei; 
- the Galactic CR flux is a power-law fitted to the AMS-02 [13, 14] $100 \mathrm{GeV}-10 \mathrm{TeV}$ data, and its knee is assumed to be either an "exponential-square" (exp2-cut) cutoff proportional to $\exp \left[-\left(E / Z R_{\text {knee }}\right)^{2}\right]$ or a change of slope (delta-slope). The slope after the knee increases by $2-\delta$, where $\delta=1 / 3$ is the slope of the diffusion coefficient. The rigidity of the knee is obtained by fitting the overall primary shape of eq. (2.1) to the KASCADEGrande data [15];

- an additional, supposedly extra-galactic ("eg”) proton component $\propto E^{-2.7}$ is added to the fitting spectrum, with its normalization as the only free parameter. The total CR flux is thus:

$$
\frac{d \Phi_{\mathrm{tot}}^{k}}{d E}=\sum_{i=p, \mathrm{He}} \frac{d \Phi_{i}^{k}}{d E}+\frac{d \Phi_{\mathrm{eg}}}{d E} \quad k=\exp 2-\mathrm{cut}, \text { delta-slope }
$$

The errors on the atmospheric neutrino flux are given by the uncertainty on the shape of the knee and by that on the normalisation and slope resulting from the fits. In table 1 we show the results of our fits.

\begin{tabular}{lrccccc}
\hline Model & \multicolumn{1}{c}{$R_{\text {knee }}$} & $N_{p}$ & $\gamma_{p}$ & $N_{\mathrm{He}}$ & $\gamma_{\mathrm{He}}$ & $N_{\mathrm{eg}}$ \\
\hline exp2-cut & $15.1 \pm 0.7 \mathrm{PV}$ & $1.5 \pm 0.2$ & $2.71 \pm 0.04$ & $1.5 \pm 0.1$ & $2.64 \pm 0.03$ & $\begin{array}{l}6.0 \pm 0.2 \\
\text { delta-slope }\end{array}$ \\
$5.8 \pm 0.6 \mathrm{PV}$ & & & & & $5.0 \pm 0.5$ \\
\hline
\end{tabular}

Table 1: The parameters of our primary CR spectrum as resulting from the fit to the AMS-02 and KASCADE-Grande data. $N_{p}$ and $N_{\mathrm{He}}$ are given in units of $10^{-7} \mathrm{GeV}^{-1} \mathrm{~m}^{-2} \mathrm{~s}^{-1} \mathrm{sr}^{-1}$ and refer to an energy of $10 \mathrm{TeV}$, while $N_{\mathrm{eg}}$ is in units of $10^{-19} \mathrm{GeV}^{-1} \mathrm{~m}^{-2} \mathrm{~s}^{-1} \mathrm{sr}^{-1}$ at $100 \mathrm{PeV}$. See [10] for more details.

\subsection{Cosmic neutrinos}

The predictions for cosmic neutrinos are much more uncertain. One of the few stable expectations is that, due to the observed three-flavor neutrino oscillations, cosmic electron, muon and tau neutrinos have to be present in similar amounts. The simplest and most popular hypothesis is that cosmic neutrinos are distributed as $E_{v}^{-\gamma}$ with $\gamma \sim 2$, at least in the observable energy range.

Such spectral index can result from hadronic collision of particles accelerated according to the diffusive shock acceleration picture, which predicts an $\propto E^{-2}$ cosmic-ray spectrum; due to the scaling associated to hadronic collisions, also the gamma-ray and neutrino spectra at the source will be power laws $\propto E^{-2}$. The abundance of target hadrons points to some dusty environment, such as some site of intense stellar formation, say, starburst and/or star-forming Galaxies. For example, the theoretical model of Loeb and Waxman [16] points to a power-law cosmic neutrino spectrum with normalization at $100 \mathrm{TeV}$ and spectral index given by:

$$
\Phi_{\text {astro }}^{\mathrm{LW}}=2 \times 10^{ \pm 0.5} \times 10^{-18} \mathrm{GeV}^{-1} \mathrm{~cm}^{-2} \mathrm{~s}^{-1} \mathrm{sr}^{-1} \quad \gamma_{\text {astro }}^{\mathrm{LW}}=2.15 \pm 0.10
$$

for an astrophysical neutrino spectrum extending above $100 \mathrm{TeV}$. 
These assumptions are consistent with the measurements of through-going muons [2] obtained by IceCube above $200 \mathrm{TeV}$ and tested by the HESE dataset [1] in the same energy region:

$$
\Phi_{\text {astro }}^{\mathrm{IC}, \mu}=0.90_{-0.27}^{+0.30} \times 10^{-18} \mathrm{GeV}^{-1} \mathrm{~cm}^{-2} \mathrm{~s}^{-1} \mathrm{sr}^{-1} \quad \gamma_{\text {astro }}^{\mathrm{IC}, \mu}=2.13 \pm 0.13
$$

Assuming $p p$-based sources of neutrinos, we expect pion decay as the main mechanism of neutrino production, thus resulting in a $\left(v_{e}: v_{\mu}: v_{\tau}\right) \simeq(1: 2: 0)$ flavor ratio at the source; due to standard three-flavor neutrino oscillations, we expect a flavor ratio at Earth of about $(1: 1: 1)$.

In order to have much more precise predictions, we define our phenomenological muon neutrino flux by combining the one as observed in [2] and that of Loeb and Waxman of eq. (2.2). We do this in the following way: 1 ) we define the combined $\gamma_{\text {astro }}$ and $\Phi_{\text {astro }}$ as the weighted average of those from [16] and [2], obtaining a muon neutrino flux of:

$$
\frac{d \Phi_{v_{\mu}}}{d E}=0.90_{-0.27}^{+0.30} \times 10^{-18}\left(\frac{E}{100 \mathrm{TeV}}\right)^{-2.14 \pm 0.08} \mathrm{GeV}^{-1} \mathrm{~cm}^{-2} \mathrm{~s}^{-1} \mathrm{sr}^{-1}
$$

2) we reproduce, using a Gaussian likelihood, the $68 \% \mathrm{CL}$ contour in the $\gamma_{\text {astro }}-\Phi_{\text {astro }}$ plane in figure 6 of [2] in order to account for the correlation (shown in figure 3 of the same paper) $\rho \sim 0.6$ between the two parameters and 3) we define the best fit astrophysical neutrino flux as the flux averaged using the same likelihood, which we also use to define the $1 \sigma$ uncertainty on the flux.

The resulting cosmic muon neutrino flux is the blue band in figure 1. It is possible to obtain the cosmic flux of $v_{e}$ and $v_{\tau}$ simply by multiplying that of muon neutrinos by $R_{e \mu}$ and $R_{\tau \mu}$; these factors are computed using the "kernel approach", which accurately embraces the strict relationship between gamma rays and neutrinos, due to the hadronic production mechanism. As shown in [17], this translates into:

$$
\frac{d \Phi_{v_{\ell}}\left(E_{v}\right)}{d E_{v}}=\int_{0}^{1} \frac{d x}{x}\left[\tilde{K}_{v_{\ell}}(x)+\tilde{K}_{\bar{v}_{\ell}}(x)\right] \frac{d \Phi_{\gamma}\left(x / E_{V}\right)}{d E} \quad \ell=e, \mu, \tau
$$

where $\tilde{K}_{v_{\ell}, \bar{v}_{\ell}}$ are kernels which account also for $v$ oscillations. For a gamma-ray flux $\propto E^{-\gamma}$ :

$$
R_{\ell \ell^{\prime}}=\frac{\zeta_{v_{\ell}}(\gamma)}{\zeta_{\ell_{\ell^{\prime}}}(\gamma)}, \text { where } \zeta_{v_{\ell}}(\gamma)=\int_{0}^{1} d x x^{\gamma-1}\left[\tilde{K}_{v_{\ell}}(x)+\tilde{K}_{\bar{v}_{\ell}}(x)\right]
$$

$R_{\ell \ell^{\prime}}$ depends negligibly on $\gamma$, and we obtained $R_{e \mu}=1.30 \pm 0.05, R_{\tau \mu}=0.92 \pm 0.04$. Note that the error on the astrophysical $v_{\mu}$ flux normalization is $30 \%$, much larger that those on $R_{e \mu}$ and $R_{\tau \mu}$, so that the relative error on the cosmic electron and tau neutrino fluxes will be $30 \%$ as well.

\section{Results and discussion}

\subsection{The components of the neutrino spectra}

In figure 1 we show the expectations for the various components of the muon neutrino flux, as well as the corresponding measurements by the IceCube Collaboration.

In figure 2 we show the prediction for the cosmic electron neutrino flux and the expectations for the other components of the electron neutrino spectrum, as well as the relevant measurement.

A few noteworthy features emerge: 1) the conventional atmospheric expectations we obtained agree well with the measurements from IceCube, 2) the region where the atmospheric and cosmic 


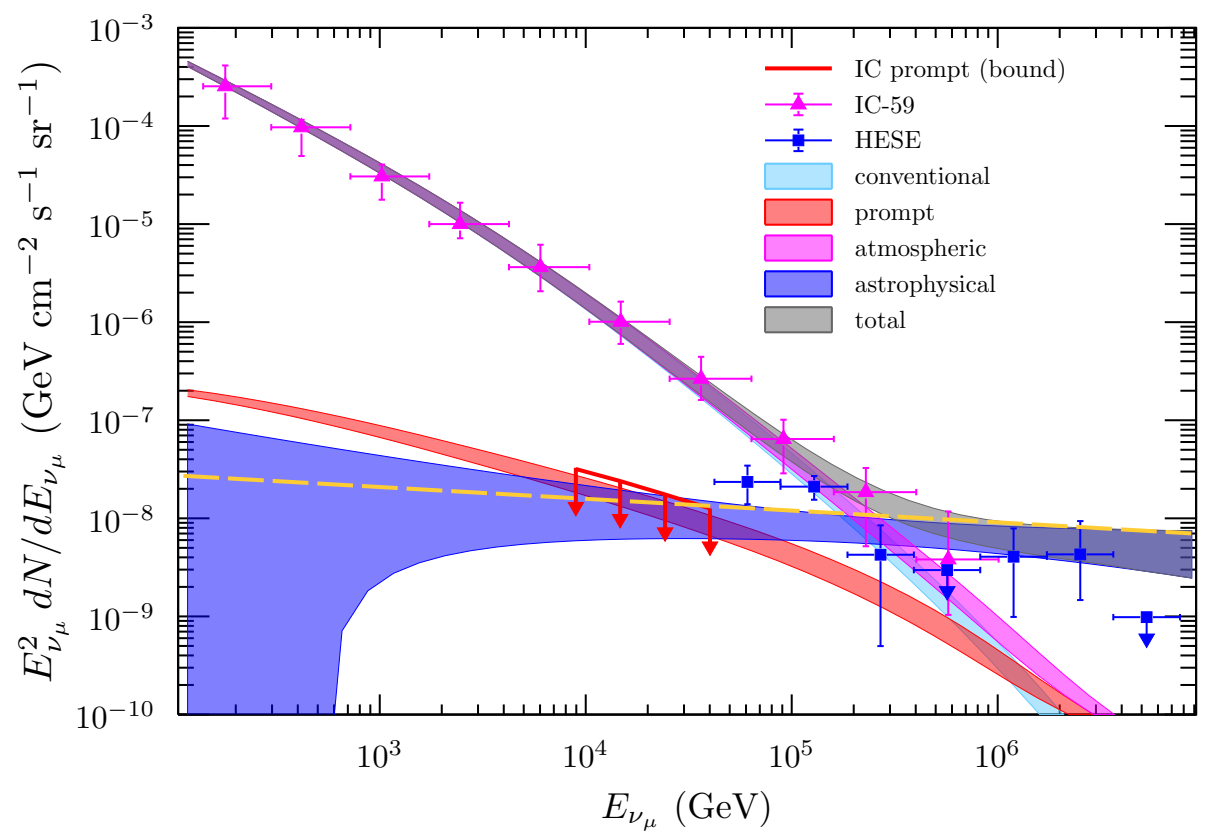

Figure 1: The components of the muon neutrino flux; also shown are the measurement of the atmospheric muon neutrino flux by IceCube [4], that of the cosmic neutrino flux with from the HESE dataset [5], the 68\% C.L. upper bound on prompt muon neutrinos in the relevant sensitivity region [2] (red line with arrows) and the upper flux limit (yellow dashed line) obtained in [6], featuring $\gamma=2.12$ and the best fit normalization + $1 \sigma$ as taken from the through-going muon analysis [2].

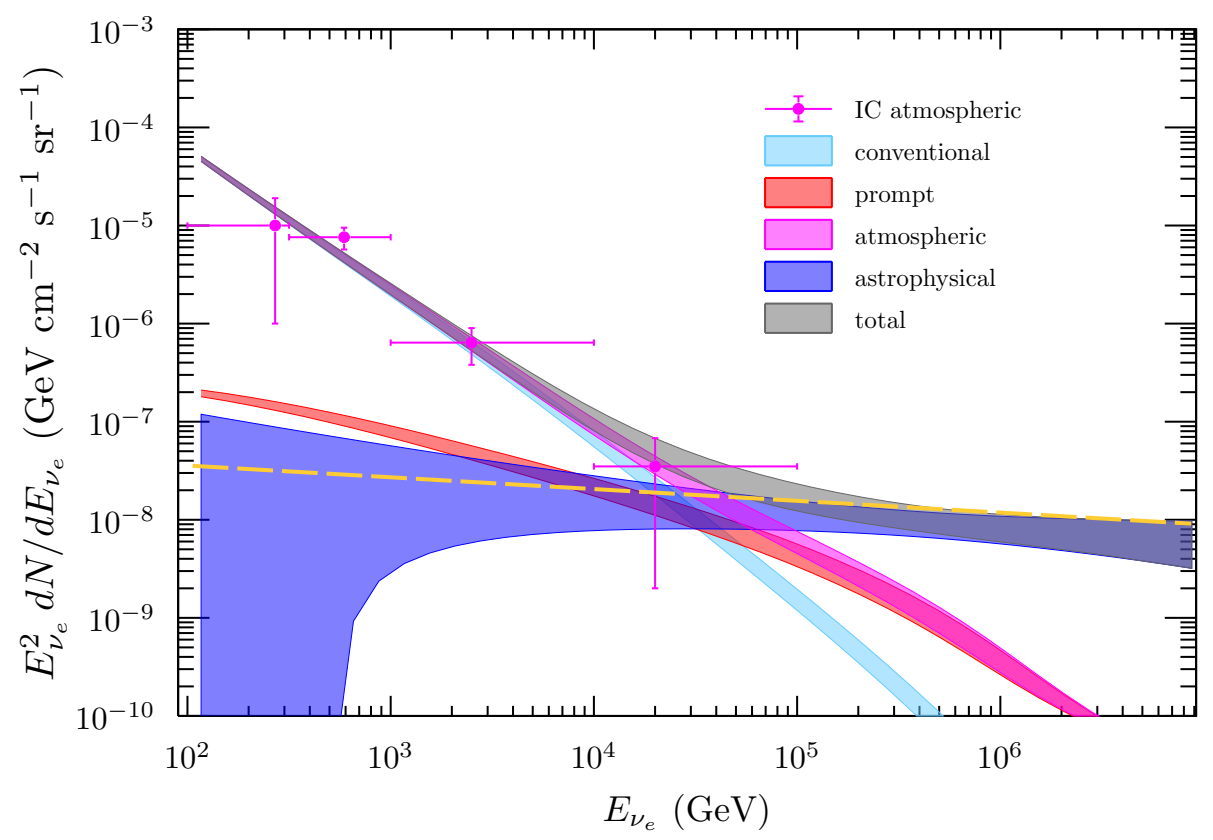

Figure 2: The predicted components of the electron neutrino flux; also shown is the measurement of the atmospheric electron neutrino flux by IceCube [3] and the upper flux limit (yellow dashed line) obtained in [6], featuring $\gamma=2.12$ and the best fit normalization $+1 \sigma$ as taken from the through-going muon analysis from [2] rescaled by $\zeta_{v_{e}} / \zeta_{v_{\mu}}$. 
components of the muon neutrino spectrum cross is around $250 \mathrm{TeV} \simeq E_{\mathrm{knee}} / 20$, 3) the prompt component is always subdominant in the $v_{\mu}$ spectrum, so that for us it is not surprising that no evidence for prompt neutrinos has been found in the through-going muons analysis, and 4) the conventional atmospheric component is $\sim 30$ times less important for $v_{e}$ 's compared to $v_{\mu}$ 's, so that the prompt component sizably contributes to the flux of $v_{e}$ for $E_{v} \geq 10 \mathrm{TeV}$.

\subsection{Is it possible to extract the prompt neutrino signal?}

\begin{tabular}{lcccc}
\hline Component & $\Gamma_{v_{e}}$ & $\Gamma_{v_{\mu}}$ & $\Gamma_{v_{\tau}}$ & $\Gamma_{\text {tot }}$ \\
\hline Conventional & $160-210$ & $420-570$ & 0 & $580-780$ \\
\hline Prompt & $20-30$ & $3-5$ & $2-3$ & $25-40$ \\
\hline Cosmic & $10-40$ & $2-6$ & $5-20$ & $15-65$ \\
\hline
\end{tabular}

Table 2: $68 \% \mathrm{CL}$ intervals relative to the yearly rate of cascade-like events in the energy range $1 \mathrm{TeV}$ and $10 \mathrm{PeV}$ in IceCube, as computed with eq. (3.1), due to the different components of the three neutrino fluxes.

Our results suggest that the best chance to detect the prompt flux of neutrinos is from atmospheric electron neutrino data for $1 \mathrm{TeV} \lesssim E \lesssim 100 \mathrm{TeV}$, assuming the possibility to discriminate the flavor of the events to some extent.

The cascade (or shower) event topology is the most interesting in this regard; cascades are produced in all cases but charged-current muon neutrino interactions, which result in tracks. The cascade sample is the one with the smallest relative contribution of muon neutrinos.

To the cascade dataset contribute: 1) atmospheric muons, 2) conventional atmospheric $v_{e}$ and $\left.v_{\mu}, 3\right)$ prompt atmospheric $v_{e}, v_{\mu}$ and $\left.v_{\tau}, 4\right)$ astrophysical $v_{e}, v_{\mu}$ and $v_{\tau}$. Therefore, provided that the contamination due to muons, conventional muon neutrinos and all-flavor astrophysical neutrinos can be subtracted, the cascade sample offers us the chance to detect prompt neutrinos.

We use the effective areas $\mathscr{A}_{v_{\ell}}(E)$ for cascade-like events given in [18] to estimate the yearly rate of cascade-like events (listed in table 2) due to all neutrino components with larger-than- $\mathrm{TeV}$ energy according to:

$$
\Gamma_{v_{\ell}}=4 \pi \times 1 \text { year } \times \int_{1 \mathrm{TeV}}^{10 \mathrm{PeV}} d E \mathscr{A}_{v_{\ell}}(E) \frac{d \Phi_{v_{\ell}}}{d E}
$$

The expected rate due to prompt neutrinos is smaller than $1 / 10$ of the conventional contribution, which is not encouraging for the search of prompt neutrinos. However, the conventional contribution could be somewhat reduced by searching for cascades coming from below and/or by using a higher energy threshold, so as to exclude most of the conventional events.

\subsection{Is there a spectral anomaly?}

In [19] and other works the spectral difference between the HESE and the through-going muons spectra measured by IceCube (evident in figure 10 from [18]) is labelled as an anomaly.

The cascade and through-going muons analyses give "complementary" indications, as cascades come from the whole sky and are due to all flavors of neutrinos, with likely a preference for electron and tau neutrinos at high energies, while through-going muons are due to muon neutrinos coming from the Northern sky. 
As can be seen from table 2, the number of prompt and cosmic signals in the cascade dataset with $E_{v}^{\text {th }} \simeq 1 \mathrm{TeV}$ are very similar to each other. Considering that they are isotropically distributed in the sky and the similar spectral index, it appears difficult to disentangle the prompt component from the astrophysical one between $1 \mathrm{TeV}$ and $100 \mathrm{TeV}$ in the cascade dataset.

The sum of the $\propto E^{-2.7}$ prompt and the $\propto E^{-2.13}$ astrophysical components may contribute significantly to the $\propto E^{-2.62}$ spectrum obtained in the cascade analysis. This is tested in figure 3, where we show the best-fit cosmic neutrino spectrum from the 1) HESE analysis [5], 2) the one from the cascade analysis [18], 3) the one from the through-going muons analysis [2] and 4) the sum of the prompt and cosmic components as computed with the model defined in section 2. We show only the $v_{e}$ flavor contribution in figure 3 because it is the one which produces the majority of the cascade events due to prompt and astrophysical neutrinos, as visible from table 2 .

While the resemblance of the spectral shape due to the sum of cosmic and prompt neutrino fluxes and the astrophysical best fits from $[18,5]$ is not perfect, the tension be-

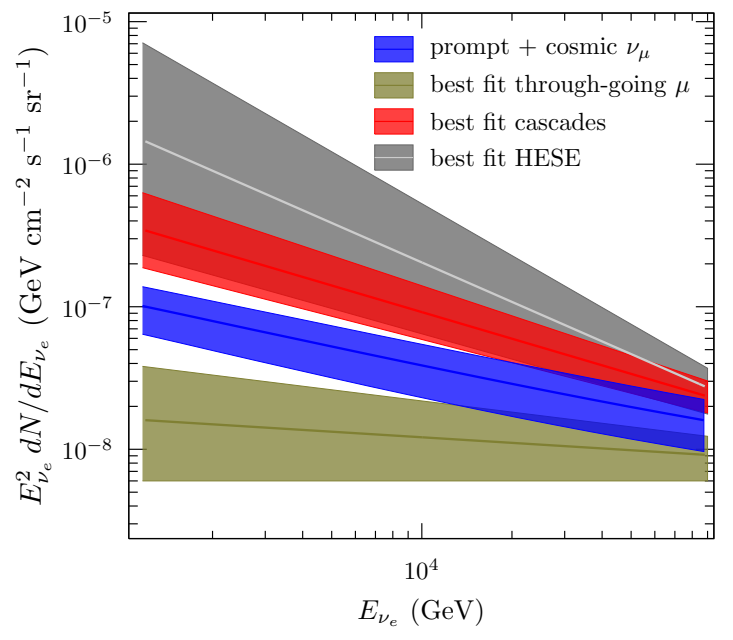

Figure 3: The sum of the prompt and cosmic components of the $v_{e}$ spectrum as computed in this work (blue band) confronted to the astrophysical neutrino fluxes resulting from fitting the cascade sample [18] (red band), from the 6-year through-going muon analysis [2] (brown band), and from the 6-year HESE analysis [5] (grey band). tween the analyses below $10 \mathrm{TeV}$ seems somewhat alleviated. From this figure, it is evident that the spectra resulting from the through-going muons and the HESE (and cascade) analyses are not compatible at low energy, which gives rise to the "spectral anomaly" of the cosmic neutrino spectrum, and that the theoretical expectations for the sum of prompt and cosmic neutrinos, instead, agrees within slightly more than $1 \sigma$ with the best-fit cosmic neutrino flux from the cascade analysis.

We conclude that the cause of the alleged spectral anomaly can be plausibly attributed to two factors: 1) a prompt component contributing to the cascade dataset in the low-energy region $\lesssim$ $100 \mathrm{TeV}, 2)$ a partial contamination of the HESE dataset due to background track events, especially at the lowest energies $\lesssim 10 \mathrm{TeV}$.

It is useful to bear in mind, that: (i) the veto system, based on the presence of muons accompanying the events with a contained vertex [20], is more effective in the energy range relevant for the search for a cosmic neutrino signal - namely, above several tens of $\mathrm{TeV}$ - rather than in the region of lower energies, most relevant for the search for prompt neutrinos; (ii) the same analysis that has obtained the cascade dataset [18] has been able to extract also a sample that is highly enriched in muons instead. Its power law description requires a slope of $2.43_{-0.30}^{+0.28}$, whose error is 3-4 times larger than for the cascade dataset and therefore is much less informative.

Other (non-exclusive) explanations of the low-energy discrepancy between the cosmic neutrino spectrum as resulting from HESE and the through-going muons analyses include: 1) a Galac- 
tic origin of the low-energy soft spectrum of HESE could be due to neutrinos, 2) other sources of extraterrestrial neutrinos, but no evidence supports either of these. Note that our proposal, concerning the role of prompt neutrinos in the cascade dataset, does not require the inclusion of hypothetical physical ingredients, and in this sense can be considered minimal.

As a final remark, we believe that great care should be taken when trying to disentangling atmospheric, and especially prompt, neutrinos from astrophysical ones between $1 \mathrm{TeV}$ and $200 \mathrm{TeV}$, and that a global analysis, adopting the same theoretical models for all datasets would be the best way to extract informations from the data.

\section{References}

[1] The IceCube Collaboration, Phys. Rev. Lett. 113 (2014) 101101 [1405. 5303].

[2] The IceCube Collaboration, Astroph. Journ. 833 (2016) 1, 3 [1607. 08006 ].

[3] The IceCube Collaboration, Phys. Rev. D91 (2015) 122004 [1504 . 03753].

[4] The IceCube Collaboration, Eur. Phys. Journ. C75 (2015) 3 [1 409 . 4535].

[5] C. Kopper on behalf of The IceCube Collaboration, in proceedings of ICRC 2017, POS (ICRC2017) 301 (2017).

[6] A. Palladino, A. Fedynitch, R. W. Rasmussen and A. M. Taylor, (2018) [1812 . 04685 ].

[7] R. Gauld, J. Rojo, L. Rottoli, S. Sarkar, J. Talbert, JHEP 2016 (2016) 2 [1511 . 0634 6].

[8] M. Benzke, M. V. Garzelli, B. A. Kniehl, G. Kramer, S. Moch, G Sigl, JHEP 2017 (2017) 12 [1705.10386].

[9] A. Bhattacharya, R. Enberg, Y. S. Jeong, C. S. Kim, M. H. Reno, I. Sarcevic, A. Stasto, JHEP 2016 (2016) 11 [1607.00193].

[10] C. Mascaretti, P. Blasi and C. Evoli, Astr. Phys. 114 (2019) 22-29 [1906. 05197].

[11] A. Fedynitch, H. Dembinski, R. Engel, T. K. Gaisser, F. Riehn and T. Stanev, Eur. Phys. Journ. Web of Conf. 99 (2015) [1503.0054 4].

[12] F. Riehn, R. Engel, A. Fedynitch, T. K. Gaisser and T. Stanev, (2015) [1510 . 0 0568].

[13] The AMS-02 Collaboration, Phys. Rev. Lett. 114 (2015) 17.

[14] The AMS-02 Collaboration, Phys. Rev. Lett. 115 (2015) 21.

[15] The KASCADE-Grande Collaboration, Phys. Rev. 87 (2013) 8 [1304 . 7114].

[16] A. Loeb and E. Waxman, JCAP 2006 (2006) 5 [astro-ph/ 0601695 ].

[17] F. L. Villante and F. Vissani, Phys. Rev. D78 (2008) 10 [0807 . 4151].

[18] The IceCube Collaboration, Phys. Rev. D99 (2019) 3 [1808 . 07629].

[19] A. Palladino, M, Spurio and F. Vissani, JCAP 2016 (2016) 12 [1610 . 07015].

[20] S. Schönert, T. K. Gaisser, E. Resconi and O. Schulz, Phys. Rev. D79 (2008) 043009 [0812 . 4308]. 